\title{
Recent boson+jet results in heavy-ion collisions with CMS
}

\author{
Inna Kucher*广 \\ Laboratoire Leprince Ringuet, France \\ E-mail: inna.kucher@cern.ch
}

\begin{abstract}
Jets produced in hard scattering processes provide an important probe of the thermodynamical and transport properties of the quark gluon plasma (QGP) created in high-energy nuclear (AA) collisions. The products of the hard scattering evolve as parton showers propagating through the medium and experience in-medium energy loss. The information on the initial jet properties can be extracted if an electroweak boson is produced together with parton in the initial hard scattering. Photon and $\mathrm{Z}$ boson-tagged measurements of the parton energy loss exploit the fact that the outgoing photon or leptons originating from $\mathrm{Z}$ boson are unmodified while traversing the QGP, and thus provide information about the momentum, direction, and flavor of the associated hard-scattered parton before it begins to shower and become quenched. In this proceeding, Z+jet and isolated $\gamma+$ jet measurements by CMS are reported.
\end{abstract}

European Physical Society Conference on High Energy Physics - EPS-HEP2019 -

10-17 July, 2019

Ghent, Belgium

\footnotetext{
* Speaker.

${ }^{\dagger}$ on behalf of the CMS collaboration
} 


\section{Introduction}

Parton energy loss has been long studied in CMS using dijet and inclusive jet measurements in $\mathrm{PbPb}$ and pp collisions $[1,2,3,4]$. However, these analyses do not have a reference which could give information about parton properties before they pass through the QGP. Boson-tagged jets, on the other hand, exploit the fact that the outgoing photon or leptons originating from $\mathrm{Z}$ boson decay are unmodified while passing through the medium, as they do not carry color charge and therefore do not interact strongly. When boson is produced in association with a parton, it is produced back to back (at leading order) having close to the same $\mathrm{p}_{\mathrm{T}}$, modulo secondary effects such as multiple scatterings of the initial partons or initial state radiation. As a result, the jet associated with the boson should have parent parton whose $\mathrm{p}_{\mathrm{T}}$, before any energy loss occurs, is well-defined by the boson tag. In addition, at LHC energies, the electroweak-boson+jet production is dominated by quark jets for jet $\mathrm{p}_{\mathrm{T}}>30 \mathrm{GeV} / \mathrm{c}$ [5], therefore providing information specifically on quark energy loss.

This proceeding summarizes selected $\mathrm{Z}+\mathrm{jet}$ and isolated $\gamma+\mathrm{jet}$ measurements performed with CMS detector, namely the boson-jet correlations [6, 7], jet fragmentation [8], and jet shapes of isolated photon-tagged jets [9]. The analyses exploit $\mathrm{PbPb}$ and pp data samples collected by $\mathrm{CMS}$ at a nucleon-nucleon center-of-mass energy of $5.02 \mathrm{TeV}$, corresponding to integrated luminosities of $404 \mu \mathrm{b}^{-1}$ and $27.4 \mathrm{pb}^{-1}$, respectively.

\section{CMS detector and object reconstruction}

The measurements are made with data collected by the CMS detector. A detailed description can be found in [10]. The silicon tracker measures the charged particles within the pseudorapidity $|\eta|<2.5$. The lead tungstate crystal electromagnetic calorimeter (ECAL), and brass and scintillator hadron calorimeter (HCAL) measure electrons, photons, and hadrons within $|\eta|<3.0$. The hadron forward calorimeter (HF) measures particle production up to $|\eta|=5.2$, the centrality is determined by the sum of the total energy deposited in HF. The centrality observable reflects the fraction of the total inelastic hadronic cross section. The most central collisions have the smallest impact parameter and the largest nuclear overlap. All these subsystems are located inside the 3.8 T superconducting solenoid. Muon energy measurement relies on a combination of inner tracking and information from the muon chambers, which are gas-ionization detectors embedded in the steel flux-return yoke outside the solenoid with the coverage up to $|\eta|<2.4$.

The event samples are selected online with dedicated lepton and photon triggers and cleaned offline to remove non-collision events. Event selection requires at least one reconstructed primary interaction vertex. Photons are reconstructed as ECAL superclusters [11]. The photon candidates are restricted to the barrel region of the ECAL, $|\eta|<1.44$, and are required to have $\mathrm{p}_{\mathrm{T}}>60 \mathrm{GeV} / \mathrm{c}$ and to pass the isolation criteria. The isolation is the additional energy in a cone of fixed radius around the direction of the reconstructed photon. It is calculated from the tracker, ECAL and HCAL with respect to the centroid of the cluster, not including the $\mathrm{p}_{\mathrm{T}}$ of the cluster and after correcting for the underlying event (UE) in $\mathrm{PbPb}$ collisions, and is required to be less than $1 \mathrm{GeV} / \mathrm{c}$. Electrons are reconstructed as ECAL superclusters matched in position and energy to tracks reconstructed in the tracker [12]. Electrons are required to have $\mathrm{p}_{\mathrm{T}}>20 \mathrm{GeV} / \mathrm{c}$ and $|\eta|<2.5$, excluding 
the ECAL barrel-endcap transition gap. In pp collisions, the electrons are selected using standard identification criteria [12], and $\mathrm{PbPb}$ collisions, the identification criteria have been optimized to compensate for the higher background levels in the calorimeters. Muons are reconstructed as segments in at least two muon detector planes and with a good-quality fit when connecting them to tracker segments. The muons are required to have $\mathrm{p}_{\mathrm{T}}>10 \mathrm{GeV} / \mathrm{c}$. The muon tracks must fall in the acceptance of the muon detectors, $|\eta|<2.4$. The same selections are applied for both $\mathrm{pp}$ and $\mathrm{PbPb}$ data. The $\mathrm{Z}$ boson candidates are defined as opposite-charge lepton pairs, with a reconstructed invariant mass in the interval $70-110 \mathrm{GeV} / \mathrm{c}^{2}$ and $\mathrm{p}_{\mathrm{T}}>40 \mathrm{GeV} / \mathrm{c}$. Jet reconstruction uses the anti- $\mathrm{k}_{\mathrm{t}}$ algorithm implemented in FASTJET [13, 14]. A small distance parameter, $\mathrm{R}=0.3$, minimizes the effects of fluctuations of the UE, dominantly formed by soft interactions of unrelated nucleon pairs in heavy ion collisions. The UE energy subtraction is performed for $\mathrm{PbPb}$ as described in [15]. Reconstructed jets are required to have $\left|\eta_{\text {jet }}\right|<1.6$ and corrected jet $\mathrm{p}_{\mathrm{T}}>30 \mathrm{GeV} / \mathrm{c}$. The resolutions of the measured jet energy and azimuthal angle in the pp samples are smeared to match those of the $\mathrm{PbPb}$ sample.

\section{Z+jet and isolated $\gamma+$ jet correlations}

$\mathrm{Z}+\mathrm{jet}$ pairs are formed from the $\mathrm{Z}$ boson and all jets in the same event. Jets reconstructed within the angular distance $\left(\Delta \mathrm{R}=\sqrt{\eta^{2}+\phi^{2}}\right)$ smaller than 0.4 from leptons are rejected to eliminate jet energy contamination by leptons coming from $Z$ boson. The jet-to- $Z \mathrm{p}_{\mathrm{T}}$ ratio reflects how much energy was lost in a jet cone, and it is measured for events with back-to-back pairs only with $\Delta \phi_{\mathrm{jZ}}>7 \pi / 8$ in order to suppress the contributions from background jets. Figure 1 shows the $\mathrm{x}_{\mathrm{jZ}}$ distributions for $\mathrm{PbPb}$ and $\mathrm{pp}$ collisions. Jet energy loss is expected to manifest itself both as a shift in the $\mathrm{x}_{\mathrm{jZ}}$ distribution and an overall decrease in the number of $\mathrm{Z}+\mathrm{jet}$ pairs as jets fall below the jet $\mathrm{p}_{\mathrm{T}}$ threshold. The average value of the transverse momentum ratio was found to be smaller in $\mathrm{PbPb}$ than in pp collisions, for all $\mathrm{Z}_{\mathrm{T}}$ ranges.

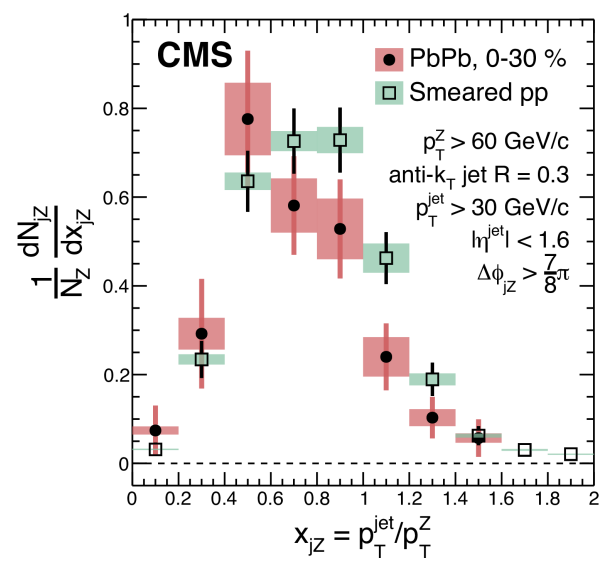

Figure 1: Distributions of the transverse momentum ratio $x_{j Z}$ between the jet and the $\mathrm{Z}$ boson with $\Delta \phi_{\mathrm{jZ}}>7 \pi / 8$. The distributions are normalized by the number of $\mathrm{Z}$ events. Vertical lines (bands) indicate statistical (systematic) uncertainties. 
$\gamma+$ jet pairs are formed from the highest $\mathrm{p}_{\mathrm{T}}$ isolated photon candidate that passes the selection criteria, and all jets in the same event. In addition to the photon and jet selections used in the study, a selection $\Delta \phi_{\mathrm{j} \gamma}>7 \pi / 8$ is applied to select back-to-back photon+jet topologies, suppressing the contributions from background jets as well as photon-multijet events. The centrality dependence of the $\mathrm{x}_{\mathrm{j} \gamma}$ distribution in $\mathrm{PbPb}$ collisions is shown in Figure 2.

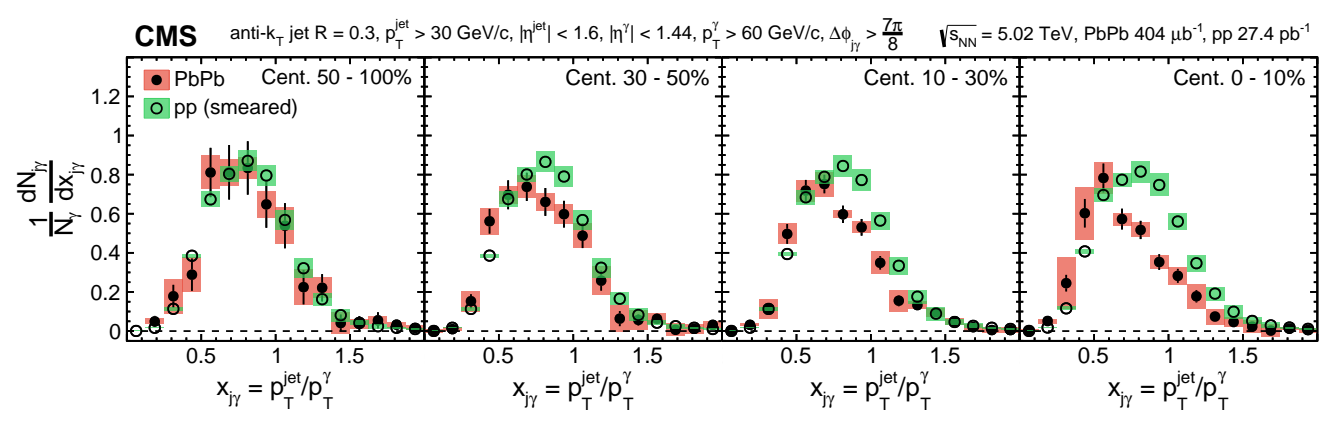

Figure 2: The centrality dependence of $\mathrm{x}_{\mathrm{j} \gamma}$ of photon+jet pairs normalized by the number of photons for $\mathrm{PbPb}$ (full markers) and smeared pp (open markers) data. The vertical lines (bands) through the points represent statistical (systematic) uncertainties.

In the most peripheral collisions (50-100\% centrality), the $\mathrm{x}_{\mathrm{j} \gamma}$ distribution agrees with the smeared pp reference data. As collisions become more central, the $\mathrm{PbPb}$ distributions shift towards lower $\mathrm{x}_{\mathrm{j} \gamma}$ and the integrals of the $\mathrm{x}_{\mathrm{j} \gamma}$ spectra become smaller. This is consistent with the expectation that a larger amount of parton $\mathrm{p}_{\mathrm{T}}$ is transported out of the jet cone as a consequence of the larger average path length that the parton needs to travel through in more central $\mathrm{PbPb}$ collisions $[16,17]$. The results are consistent between $\mathrm{Z}+$ jet and $\gamma+$ jet measurements.

\section{Jet fragmentation and jet shapes of isolated photon-tagged jets}

Jet fragmentation patterns are studied in $\gamma+$ jet system, where $\gamma+$ jet pairs and charged-particle tracks are used to determine the fragmentation functions. $\gamma+$ jet pairs are constructed in the same way as described in the previous section. Track $\mathrm{p}_{\mathrm{T}}$ is required greater than $1 \mathrm{GeV} / \mathrm{c}$ and tracks are required to fall within a cone of the radius $R=0.3$ around the jet direction.

Photon-tagged fragmentation functions are defined as $\xi^{\text {jet }}=\ln \frac{-\left|\overrightarrow{\mathrm{p}}^{\text {jett }}\right|^{2}}{\overrightarrow{\mathrm{p}}^{\text {trk. }} \cdot \overrightarrow{\mathrm{p}}^{\text {jet }}}$ and $\xi_{\mathrm{T}}^{\gamma}=\ln \frac{-\left|\overrightarrow{\mathrm{p}}_{\mathrm{T}}^{\gamma}\right|^{\gamma}}{\overrightarrow{\mathrm{p}}_{\mathrm{T}}^{\text {trkr }} \cdot \overrightarrow{\mathrm{p}}_{\mathrm{T}}^{\gamma}}$. The $\xi^{\text {jet }}$ gives the fragmentation pattern with respect to $\mathrm{p}_{\mathrm{T}}$ of the reconstructed jet [18], and can be compared directly with results obtained using a dijet sample [4]. The $\xi_{\mathrm{T}}^{\gamma}$ variable is used to characterize the fragmentation pattern with respect to the $\mathrm{p}_{\mathrm{T}}$ of the initial parton before any energy loss occurred. The latter is shown in Figure 3.

The distribution of $\xi_{\mathrm{T}}^{\gamma}$ in the peripheral $\mathrm{PbPb}$ events is consistent with those in pp data. In more central collisions, an enhancement is observed in the $\mathrm{PbPb}$ data relative to pp data in the $\xi_{\mathrm{T}}^{\gamma}>3$ region, which corresponds to $\mathrm{p}_{\mathrm{T}}^{\mathrm{trk}}<3 \mathrm{GeV} / \mathrm{c}$ for $\mathrm{p}_{\mathrm{T}}^{\gamma}=60 \mathrm{GeV} / \mathrm{c}$ and $\Delta \phi \simeq \pi$ between the track and the photon. The magnitude of this enhancement increases as the $\mathrm{PbPb}$ collisions become more central. A suppression of the $\xi_{\mathrm{T}}^{\gamma}$ distribution in the most central $\mathrm{PbPb}$ collisions is observed for $0.5<\xi_{\mathrm{T}}^{\gamma}<3$, which corresponds to $3<\mathrm{p}_{\mathrm{T}}^{\mathrm{trk}}<36 \mathrm{GeV} / \mathrm{c}$ for $\mathrm{p}_{\mathrm{T}}^{\gamma}=60 \mathrm{GeV} / \mathrm{c}$ and $\Delta \phi \simeq \pi$ between 


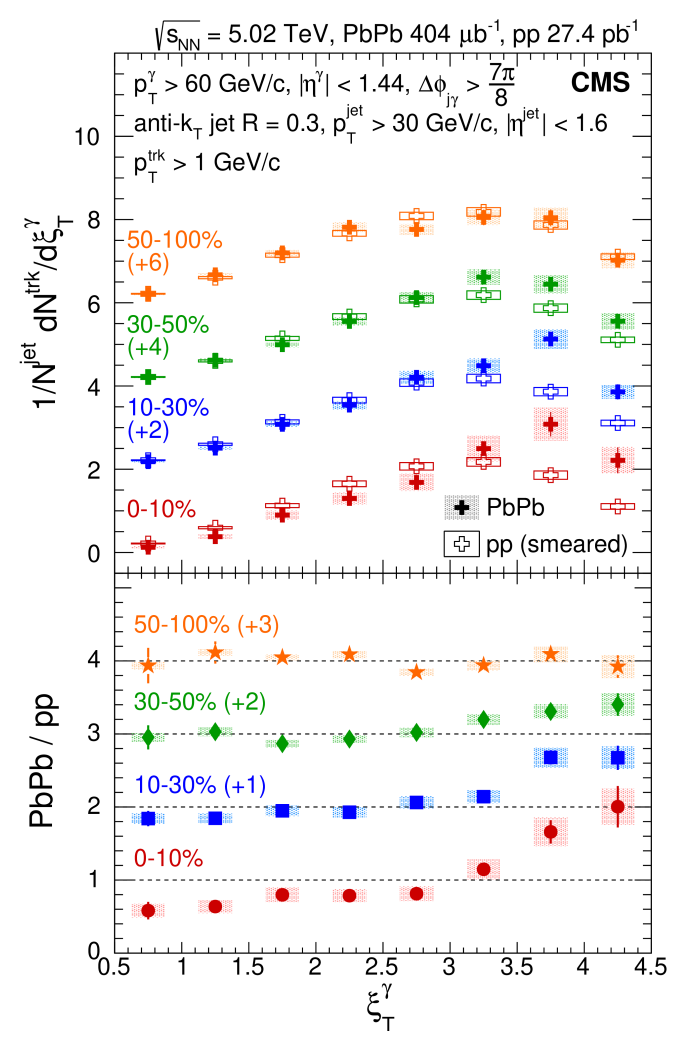

Figure 3: The centrality dependence of the $\xi_{\mathrm{T}}^{\gamma}$ distribution for jets associated with an isolated photon for $\mathrm{PbPb}$ (full crosses) and $\mathrm{pp}$ (open crosses) collisions. The pp results are smeared for each $\mathrm{PbPb}$ centrality bin, and data for each centrality bin are shifted vertically as indicated. Bottom: The ratios of the PbPb over smeared pp distributions. The vertical bars through the points represent statistical uncertainties, while the colored boxes indicate systematic uncertainties.

the track and the photon. This pattern of suppression and enhancement is a direct evidence for energy loss by high- $\mathrm{p}_{\mathrm{T}}$ partons as they traverse the medium $[19,20]$.

The differential jet shape for jets associated with an isolated photon gives information of how the $\mathrm{p}_{\mathrm{T}}$ of a jet is distributed (over charged particles) in a direction transverse to the jet axis, and it is defined as $\rho(\mathrm{r})=\frac{1}{\delta \mathrm{r}} \frac{\sum_{\text {jets }} \sum_{\mathrm{r}_{\mathrm{a}}<\mathrm{r}<r_{\mathrm{b}}}\left(\mathrm{p}_{\mathrm{T}}^{\text {trk }} / \mathrm{p}_{\mathrm{T}}^{\text {jet }}\right)}{\sum_{\text {jets }} \sum_{0<\mathrm{r}<\mathrm{r}_{\mathrm{f}}}\left(\mathrm{p}_{\mathrm{T}}^{\text {trk }} / \mathrm{p}_{\mathrm{T}}^{\text {jet }}\right)}$, where $\mathrm{r}=\sqrt{\left(\eta^{\text {jet }}-\eta^{\text {trk }}\right)^{2}+\left(\phi^{\text {jet }}-\phi^{\text {trk }}\right)^{2}}$ is the distance between the track and the jet axis in $\eta-\phi$ plane, $\delta \mathrm{r}=\mathrm{r}_{\mathrm{b}}-\mathrm{r}_{\mathrm{a}}$ is the width of the ring-shaped area of inner and outer radii $r_{a}$ and $r_{b}$ with respect to the jet axis. The distribution is normalized such that the integral inside the range $0<\mathrm{r}<0.3$ is equal to 1 . The jet shape is constructed using charged particles with transverse momentum $\mathrm{p}_{\mathrm{T}}^{\mathrm{trk}}>1 \mathrm{GeV} / \mathrm{c}$, for jets with $\mathrm{p}_{\mathrm{T}}>30 \mathrm{GeV} / \mathrm{c}$, which are associated with an isolated photon with $\mathrm{p}_{\mathrm{T}}^{\gamma}>60 \mathrm{GeV} / \mathrm{c}$.

The upper panel of Figure 4 shows the differential jet shape or both $\mathrm{PbPb}$ and pp collisions, and PYTHIA simulation. The ratio of $\mathrm{PbPb}$ to $\mathrm{pp}$ (simulated to $\mathrm{pp}$ ) data distributions are shown in the lower panel.

The distribution from the most peripheral (50\%-100\%) $\mathrm{PbPb}$ collisions is consistent with that in pp data, a modification of the jet shape in $\mathrm{PbPb}$ collisions is observed in more central events. 


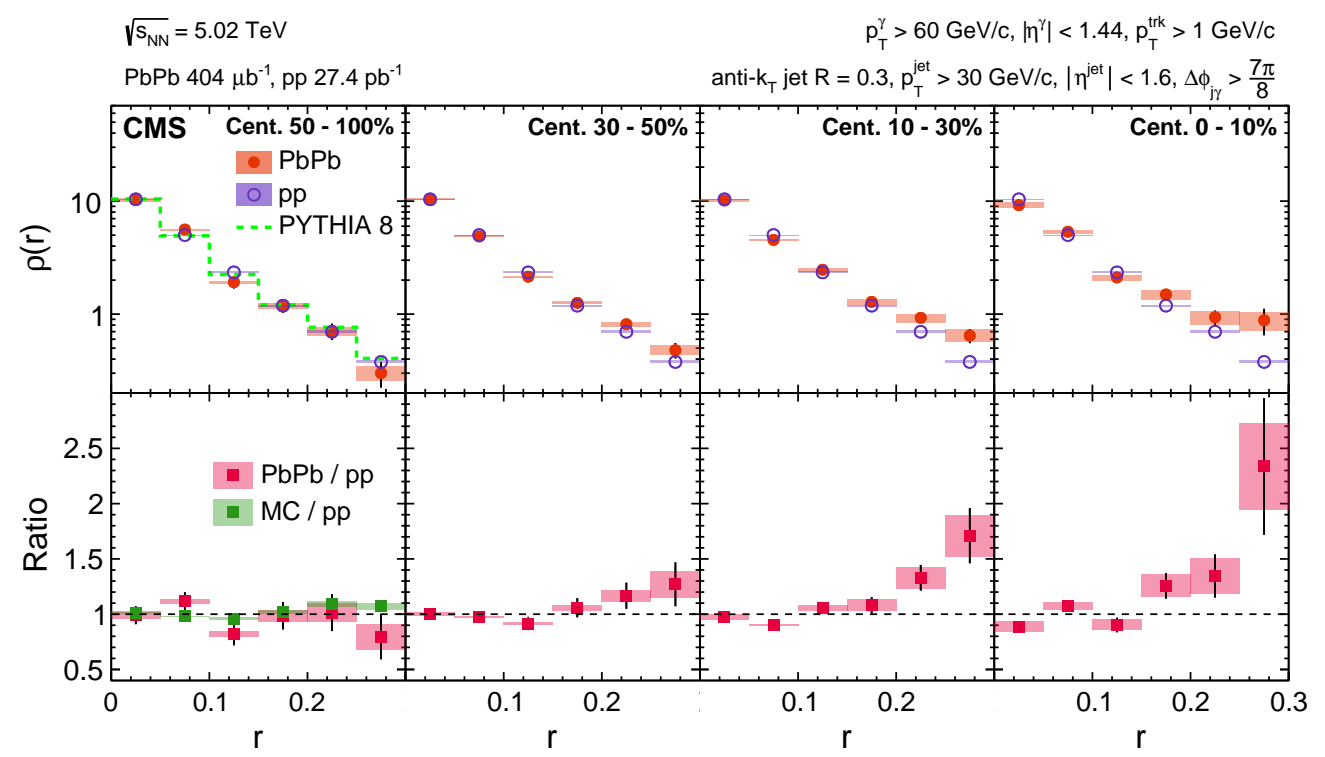

Figure 4: Upper: The differential jet shape, $\rho(\mathrm{r})$, for jets associated with an isolated photon for (from left to right) $50-100 \%, 30-50 \%, 10-30 \%, 0-10 \% \mathrm{PbPb}$ (full circles), and pp (open circles) collisions and from PYTHIA simulation (histogram). Lower: The ratios of the $\mathrm{PbPb}$ and pp distributions. For the pp results, the ratios is to the PYTHIA distribution. The vertical lines through the points represent statistical uncertainties, the shaded colored boxes indicate the total systematic uncertainties in data.

The $0 \%-10 \% \mathrm{PbPb} \rho(\mathrm{r})$ is enhanced for the distance between the track and the jet axis $\mathrm{r}>0.15$. No significant suppression is seen at intermediate $r$. The modifications demonstrate that for hard scatterings that predominantly produce quarks with similar momentum distributions in $\mathrm{pp}$ and $\mathrm{PbPb}$ collisions, as identified by the photon tag, the jet momentum is distributed at greater radial distance in $\mathrm{PbPb}$ collisions. This observation can be interpreted as a direct observation of jet broadening in the QGP.

\section{Summary}

This proceeding briefly summarizes CMS measurements of the jet-to-boson $\mathrm{p}_{\mathrm{T}}$ balance, jet fragmentation functions, and jet radial profile with $Z+j e t$ and $\gamma+$ jet configurations. These measurements show the in-medium parton shower modifications for events with well-defined initial parton kinematics, and constitutes well-controlled reference for testing theoretical models of the parton's passage through the QGP. More details, results and comparisons to theoretical models can be found in the original CMS papers $[6,7,8,9]$.

\section{References}

[1] CMS Collaboration, Measurement of inclusive jet cross sections in pp and PbPb collisions at $\sqrt{\mathrm{s}_{\mathrm{NN}}}=2.76 \mathrm{TeV}$, Phys. Rev. C 96, 015202 (2017).

[2] CMS Collaboration, Measurement of jet fragmentation into charged particles in $p p$ and $\mathrm{PbPb}$ collisions at $\sqrt{\mathrm{s}_{\mathrm{NN}}}=2.76 \mathrm{TeV}$, J. High Energy Phys. 10 (2012) 087. 
[3] CMS Collaboration, Modification of jet shapes in PbPb collisions at $\sqrt{\mathrm{s}_{\mathrm{NN}}}=2.76 \mathrm{TeV}$, Phys. Lett. B 730,243 (2014).

[4] CMS Collaboration, Measurement of jet fragmentation in PbPb and pp collisions at $\sqrt{\mathrm{s}_{\mathrm{NN}}}=2.76$ TeV, Phys. Rev. C 90, 024908 (2014).

[5] U. A. Wiedemann, in Relativistic Heavy Ion Physics, edited by R. Stock (Landolt-Börnstein/SpringerMaterials, New York, 2010), Vol. 23, p. 521.

[6] CMS Collaboration, Study of jet quenching with isolated-photon + jet correlations in PbPb and pp collisions at $\sqrt{\mathrm{s}_{\mathrm{NN}}}=5.02 \mathrm{TeV}$, Physics Letters B 785 14-39 (2018).

[7] CMS Collaboration, Study of Jet Quenching with Z+jet Correlations in Pb-Pb and pp Collisions at $\sqrt{\mathrm{s}_{\mathrm{NN}}}=5.02 \mathrm{TeV}$, Phys. Rev. Lett. 119, 082301 (2017).

[8] CMS Collaboration, Observation of Medium-Induced Modifications of Jet Fragmentation in Pb-Pb Collisions at $\sqrt{\mathrm{s}_{\mathrm{NN}}}=5.02 \mathrm{TeV}$, Phys. Rev. Lett. 121, 242301 (2018).

[9] CMS Collaboration, Jet Shapes of Isolated Photon-Tagged Jets in Pb-Pb and pp Collisions at $\sqrt{\mathrm{s}_{\mathrm{NN}}}=5.02 \mathrm{TeV}$, Phys. Rev. Lett. 122, 152001 (2019).

[10] CMS Collaboration, The CMS experiment at the CERN LHC, JINST 3 S08004 (2008).

[11] CMS Collaboration, Performance of photon reconstruction and identification with the CMS detector in proton-proton collisions at $\sqrt{\mathrm{s}}=8 \mathrm{TeV}$, JINST $10 \mathrm{P} 08010$ (2015).

[12] CMS Collaboration, Performance of electron reconstruction and identification with the CMS detector in proton-proton collisions at $\sqrt{\mathrm{s}}=8 \mathrm{TeV}$, JINST 10 P06005 (2015).

[13] M. Cacciari, G. P. Salam, and G. Soyez, The anti- $\mathrm{k}_{\mathrm{t}}$ jet clustering algorithm, J. High Energy Phys. 04 (2008) 063.

[14] M. Cacciari, G. P. Salam, and G. Soyez, FastJet user manual, Eur. Phys. J. C 72, 1896 (2012).

[15] Olga Kodolova, I. Vardanian, A. Nikitenko, and A. Oulianov, The performance of the jet identification and reconstruction in heavy ions collisions with CMS detector, Eur. Phys. J. C 50, 117 (2007).

[16] ATLAS Collaboration, Measurement of the jet radius and transverse momentum dependence of inclusive jet suppression in lead-lead collisions at $\sqrt{\mathrm{s}_{\mathrm{NN}}}=2.76 \mathrm{TeV}$ with the ATLAS detector, Phys. Lett. B 719 (2013) 220.

[17] CMS Collaboration, Measurement of transverse momentum relative to dijet systems in $\mathrm{PbPb}$ and $\mathrm{pp}$ collisions at $\sqrt{\mathrm{s}_{\mathrm{NN}}}=2.76$, J. High Energy Phys. 01 (2016) 006.

[18] F. Arleo, P. Aurenche, Z. Belghobsi, and J.-P. Guillet, Photon tagged correlations in heavy ion collisions, J. High Energy Phys. 11 (2004) 009.

[19] X.-N. Wang, Z. Huang, and I. Sarcevic, Jet Quenching in the Opposite Direction of a Tagged Photon in High-Energy Heavy Ion Collisions, Phys. Rev. Lett. 77, 231 (1996).

[20] X.-N. Wang and Z. Huang, Medium-induced parton energy loss in $\gamma+$ jet events of high-energy heavy-ion collisions, Phys. Rev. C 55, 3047 (1997). 